\title{
Antioxidant Activity of Garcinia cf bancana Miq
}

\author{
Sri Hartati ${ }^{1}$, Triyem ${ }^{2}$ and Herry Cahyana ${ }^{2}$ \\ Research Center for Chemistry, Indonesia Institute of Sciences, \\ Kawasan Puspiptek Serpong Tangerang. \\ Department of Chemistry, Indonesia University. UI- Depok
}

\begin{abstract}
Guttiferron $F$ and essential oil were isolated from extract $n$-hexane of Garcinia of bancana Miq. Antioxidant activity was done by DPPH method to $n$-hexane, methanol, ethyl acetate, $n$-butanol extracts and Guttiferron $F$, showed activity with $I C_{50} 24.5,22.4,29.2,37.6$ and $25.8 \mu \mathrm{g} / \mathrm{mL}$ respectively. The structure elucidation of Guttiferon F based on spectroscopy data of IR, NMR of $\mathrm{IH}$ and ${ }^{13} \mathrm{C}$.
\end{abstract}

Keywords: Antioxidant, Garcinia of bancana Miq., Essential oil and Guttiferon-F.

\section{INTRODUCTION}

Garcinia $C$ f bancana Miq similarity with $G$. bacana Miq belonging to the Guttiferae family is distributed throughout Southern Thailand, Malaysia and Indonesia (Withmore, 1973). Some of Garcinia species used as traditional medicines as G. picroriza aand G. atroviridis used for fever (Burkill, 1935), G. mangostana used as antidhiarea and anti inflammatory (Ballasubranian et al., 1988), G. dulcis used as lymphatitis, struma and parolitis (Inuma et al, 1996). Some compounds of Garcinia species have potentially bioactivity as antioxidant (Yamaguchi et al., 2000; Terashima et al., 2002; Anne et al. 2004 and Liao et al., 2005), antibacterial and antifungal (Permana et al., 2001; Vancharin et al., 2005a, 2005b; Suksamrarn et al., 2003; Sudpodma et al., 2005 and Quan-Bin et al., 2005), cytotoxic ( Odile et al., 2000; Xu et al., 2000; Chi-Kuan et al., 2002; Quan-Bin et al., 2006; Suksamrarn et al., 2006), antimalarial (Likhitwitiawuid et al., 1998a, 1998 b; Tona et al., 2004) and HIV-I inhibitory activities (Chen et al., 1996). In search to investigation antioxidant from natural product, have been done antioxidant activity assay with radical scavenger (DPPH) method of $n$-hexane, methanol, ethyl acetate and $n$ butanol extracts and isolation of main active compound of bark G. $c f$ bancana Miq.

\section{METHODS}

\section{Plant material}

The barks of Garcinia cf bancana was collected from village Kalapangan, district Sebangau Palangkaraya Central Kalimatan. Species determine was conducted by Ismail and voucher specimens deposited at the Herbarium Bogorience of Research Center for Biology-LIPI. Bogor.

\section{General methods}

Melting point was measured on Fisher Scientific Apparatus. IR spectra was obtained with a Shimadzu Perkin Elmer 16 PC-FT-IR Prestige 21 Spectrometer. 1D and 2 D NMR were performed on I NOVA Plus, Unity NMR $500 \mathrm{MHz}$ spectrometer with tetra-methyl-sylane (TMS) as internal standard. LC-MS Mariner Biospectrometry, LC: Hitachi L 6200, System FSI (Electrospray Ionization), Positive ion mode, column C-18 Supelco, column length: $150 \mathrm{~mm}$, ID: $2 \mathrm{~mm}$, particle size: $5 \mathrm{um}$. GC-MS Shimadzu QP 5050A, detector DDSMS, temperature column $60^{\circ} \mathrm{C}$; temperature detector $300^{\circ} \mathrm{C}$, temperature injector $310^{\circ} \mathrm{C}$, time analyze $30 \mathrm{~min}$., volume injection $0.2 u \mathrm{~L}$. Silica gel (65- 250 mesh and 230 - 400 mesh) from E-Merck, Sephadex LH-20 from Amersham Bioscience. DPPH 1,1-diphenyl-2pycrylhidrayl was purchased from Sigma Chemical Co. 


\section{Extraction and Isolation}

The ground dried stem barks of $G$. $c f$ bancana Miq $(4.58 \mathrm{Kg})$ was percolated with $n$ hexane $(3 \times 5 \mathrm{~L})$ at room temperature, the solvent was evaporated in vacuum to afford a hexane extract $(190 \mathrm{~g})$. The residue continued to percolated with methanol $(4 \times 5 \quad$ L) at room temperature, the solvent $(\mathrm{MeOH})$ was evaporated in vacuum to afford $\mathrm{MeOH}$ extract $(420 \mathrm{~g})$. The $\mathrm{MeOH}$ extract $(100 \mathrm{~g})$ then suspended in $\mathrm{H}_{2} \mathrm{O}(1$ L) and portioned with ethyl acetate $(3 \times 1 \mathrm{~L})$ the separated fraction ethyl acetate from $\mathrm{H}_{2} \mathrm{O}$ and evaporated in vacuum to afford ethyl acetate extract $(10 \mathrm{~g})$. To the water fraction added $n$ butanol $(3 \times 1 \mathrm{~L})$ to portioned and the separated fraction $n$ - butanol from $\mathrm{H}_{2} \mathrm{O}$ and evaporated in vacuum to afford $n$ - butanol extract $(40 \mathrm{~g})$. The $n$ hexane extract $(37 \mathrm{~g})$ was subjected to silica gel flash column chromatography (CC: $\Phi 5 \mathrm{~cm} ;<45$ $u \mathrm{~m} ; 50 \mathrm{~g})$ using gradient mixture of $n$-hexaneethyl acetate (0-100 \%) as mobile phases, affording four fractions (FA-FD). FA fraction is colorless essential mixture oil $(1.64 \mathrm{~g})$ was analyzed by GC-MS. FD fraction was subjected to sephadex LH-20 column using mixture of $\mathrm{CH}_{2} \mathrm{Cl}_{2}$ : $\mathrm{MeOH}(1: 1)$ as mobile phase, resulting three fractions $\left(\mathrm{FD}_{1}-\mathrm{FD}_{3}\right)$, the second fraction (200 $\mathrm{mg}$ ) was further purified by preparative thin layer chromatography (TLC) yielded compound 5 (10.2 $\mathrm{mg}$ ).

Each extracts and compound $\mathbf{5}$ was tested for antioxidant properties base on scavenging activity study using stable 1,1- diphenyl-2pycrylhidrazyl (DPPH) free radical.

\section{Scavenging Activity of DPPH radicals}

The free radical-scavenging activity (Molyneux, 2004) method was tested as bleaching of stable 1,1- diphenyl-2-pycrylhidrazyl radical. In its radical from $\mathrm{DPPH}^{\circ}$ has an absorption band at $520 \mathrm{~nm}$ (dark purple), which disappear upon reduction by antiradical compound. The principle reaction as bellow: (Figure. 1).

The reaction mixture containing test sample $25,125,250$ and $500 \mu \mathrm{L}$ respectively (1000 ppm in $\mathrm{MeOH})$ and $500 \mu \mathrm{L}$ of DPPH $(0.5$ $\mathrm{mM}$ in the $\mathrm{MeOH}$ ) are added to each samples and dilute in $\mathrm{MeOH}$ until $2.5 \mathrm{~mL}$ and incubated at $37^{\circ} \mathrm{C}$ for $30 \mathrm{~min}$. The absorbance was measured at $515 \mathrm{~nm}$. Percent radical scavenging activity was measured by comparison with $\mathrm{MeOH}$ containing control. $\mathrm{IC}_{50}$ values represent of compound (samples) to scavenge $50 \%$ of DPPH radical. Cuercetine was used as positive control.

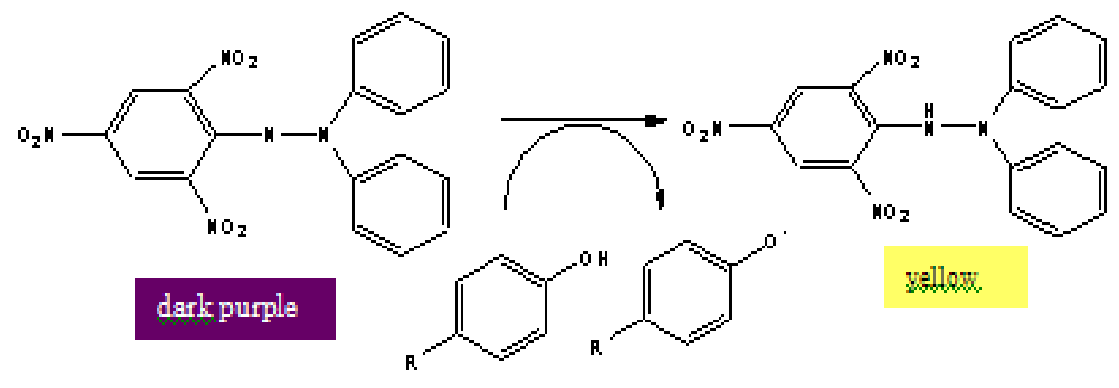

Figure I. The principle reaction of DDPH

\section{RESULTS}

From the analysis of FA (essential oil) with GCMS resulted mixture of compounds:

a. Copaene 1 with retention time $10.42 \mathrm{~min}$, with $\mathrm{Mr}=204$, molecule formula $\mathrm{C}_{15} \mathrm{H}_{24}$ and with percent SI (similarity) is $96 \%$.

b. 4,7-methanoazulene $\mathbf{2}$ have retention time $10.88 \mathrm{~min}$, with $\mathrm{Mr}=204$, molecule formula $\mathrm{C}_{15} \mathrm{H}_{24}$ and percent $\mathrm{SI}$ is $91 \%$. c. $\gamma$-cadienene 3 with retention time $12.58 \mathrm{~min}$ with $\mathrm{Mr}=204$ and molecule formula $\mathrm{C}_{15}$ $\mathrm{H}_{24}$, have percent $\mathrm{SI}$ is $95 \%$.

d. Cariofilen oxide $\mathbf{4}$ have retention time 13.99 min with $\mathrm{Mr}=220$ and molecule formula $\mathrm{C}_{15} \mathrm{H}_{24} \mathrm{O}$, with percent $\mathrm{SI}$ is $97 \%$. The structures 1, 2, 3 and $\mathbf{4}$ are (Figure. 2): 


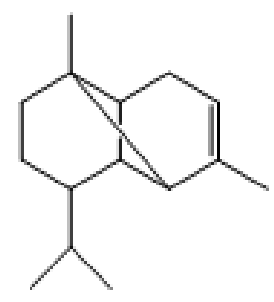

1. Copaene

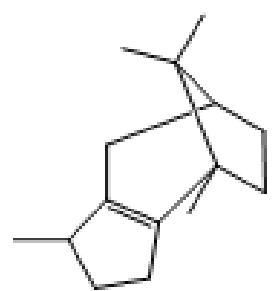

2. 4,7-methanoazulene<smiles>C=C1CCC(C(C)C)C2C=C(C)CCC12</smiles>

3. $\gamma$-cadienene

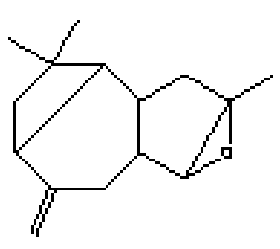

4. Cariofilen oxide

Figure 2. Four essentials structure I: 2; 3 and 4

Compound 5: was isolated as yellow powder (10.2 mg), melting point $133-135{ }^{\circ} \mathrm{C}$, having the $\mathrm{MH}^{+}$at the $\mathrm{m} / \mathrm{z}=603$. Melting point $133-135^{\circ} \mathrm{C}$. The IR (in $\mathrm{KBr}$ ) spectrum showed strong bands (3406-3257 $\left.\mathrm{cm}^{-1}\right)$ and sharp bands at $1726 \mathrm{~cm}^{-1}$, $1638 \mathrm{~cm}^{-1}, 1600 \mathrm{~cm}^{-1}$. The ${ }^{1} \mathrm{H}-\mathrm{NMR}$ data (in $\left.\mathrm{C}_{3} \mathrm{D}_{6} \mathrm{O}, 500 \mathrm{MHz}\right)$ showed chemical shift $\left(\delta^{1} \mathrm{H}\right.$, ppm) $0.99(3 \mathrm{H}, s) ; 1.17(3 \mathrm{H}, s) ; 1.45(3 \mathrm{H}, s) ; 1.49$ $(3 \mathrm{H}, s) ; 1.59(3 \mathrm{H}, s) ; 1.60(3 \mathrm{H}, s) ; 1.62(3 \mathrm{H}, s)$; $1.63(3 \mathrm{H}, s) ; 1,68(3 \mathrm{H}, s) ; 1.69(3 \mathrm{H}, s) ; 1.93(2 \mathrm{H}$, $d d, J=12.85 ; 6.1 \mathrm{~Hz}) ; 2.01(\mathrm{~m}) ; 2.02(\mathrm{~m}) ; 2.20(d$, $J=14.50 \mathrm{~Hz}) ; 2.69(\mathrm{~m}) ; 2.70(\mathrm{~m}) ; 4.50(\mathrm{~s}) ; 4,51$ $(s) ; 4.93(1 \mathrm{H}, t) ; 5.02(1 \mathrm{H}, t) ; 5.09(1 \mathrm{H}, b) ; 6.71$ $(1 \mathrm{H}, d, J=7.30 \mathrm{~Hz}) ; 7.03(1 \mathrm{H}, d, J=7.35 \mathrm{~Hz}) ; 7.20$ $(s, 1 \mathrm{H})$. The ${ }^{13} \mathrm{C}$-NMR $\left(125 \mathrm{MHz}\right.$, in $\mathrm{C}_{3} \mathrm{D}_{6} \mathrm{O}$ and DEPT) data showed chemical shift $\left(\delta^{13} \mathrm{C}\right.$, ppm) $17.90\left(\mathrm{CH}_{3}\right) ; 18.12\left(\mathrm{CH}_{3}\right) ; 18.18\left(\mathrm{CH}_{3}\right) ; 18.38$
$\left(\mathrm{CH}_{3}\right) ; 23.04\left(\mathrm{CH}_{3}\right) ; 25.74\left(\mathrm{CH}_{3}\right) ; 25.82\left(\mathrm{CH}_{3}\right)$; $26.16\left(\mathrm{CH}_{3}\right) ; 26.59\left(\mathrm{CH}_{2}\right) ; 27.21\left(\mathrm{CH}_{2}\right) ; 27.33$ $\left(\mathrm{CH}_{3}\right) ; 32.75(\mathrm{CH}) ; 37.13\left(\mathrm{CH}_{2}\right) ; 42.92\left(\mathrm{CH}_{2}\right)$; $44.40(\mathrm{CH}) ; 48.88(\mathrm{C}) ; 47.60(\mathrm{C}) ; 49.50(\mathrm{C}) ; 69.00$ (C); $112.05\left(\mathrm{CH}_{2}\right) ; 114.69(\mathrm{CH}) ; 116.91(\mathrm{CH})$; $116.91(\mathrm{C}) ; 122.07(\mathrm{CH}) ; 124.03(\mathrm{CH}) ; 125.09$ $(\mathrm{CH}) ; 125.89(\mathrm{CH}) ; 131.38(\mathrm{C}) ; 131.46(\mathrm{C}) ; 132.29$ (C); 132.29 (C); $145.11(\mathrm{C}-\mathrm{OH}) ; 149.40(\mathrm{C}-\mathrm{OH})$; 150.31 (C); $171.50(\mathrm{C}-\mathrm{OH}) ; 196.50(\mathrm{C}=\mathrm{O}) ; 211.00$ $(\mathrm{C}=\mathrm{O})$. HMQC (Heteronuclear Multiple Quantum Coherence) and HMBC (Heteronuclear Multiple Bond Corelation) data of compound $\mathbf{5}$ showed in Table 2.

The Scavenging Activity of DPPH radicals result showed in Table 1

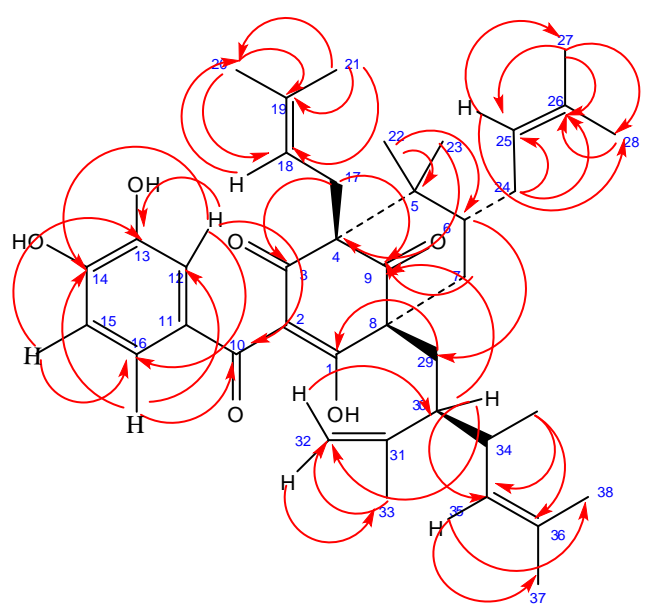

Figure 3. Correlation $\mathrm{HMBC}$ of compund 5<smiles>C=C(C)[C@H](CC=C(C)C)C[C@]1(C)C(O)=C(C(=O)c2ccc(O)c(O)c2)C(=O)[C@@]2(CC=C(C)C)C(=O)[C@]1(C)C[C@@H](CC=C(C)C)C2(C)C</smiles>

Figure 4. Guttiferon $F$ 
Table I. The IC 50 antioxidant of extracts and compound 5

\begin{tabular}{|c|c|}
\hline Sample & $I_{50} \mu \mathrm{g} / \mathrm{mL}$ \\
\hline Cuercetine & 10.09 \\
\hline$n$-hexana exstract & 24,50 \\
\hline Methanol exstract & 22,35 \\
\hline Ethyl acetate exstract & 29,17 \\
\hline n-butanol exstract & 37.56 \\
\hline Compound $\mathbf{5}$ (Guttiferon F) & 25.79 \\
\hline
\end{tabular}

\section{DISCUSSION}

\section{Essential oil}

From the analysis with GC-MS of FA (essential oil) resulted a mixture of compounds Copaene $\mathrm{Mr}=204$, molecule formula $\left(\mathrm{C}_{15} \mathrm{H}_{24}\right)$ with percent SI (similarity) is 96\%. 1; 4,7methanoazulene, with $\mathrm{Mr}=204$, molecule formula $\mathrm{C}_{15} \mathrm{H}_{24}$ and percent $\mathrm{SI}$ is $91 \%$. 2, $\gamma$-cadienene with $\mathrm{Mr}=204$ and molecule formula $\mathrm{C}_{15} \quad \mathrm{H}_{24}$, have percent $\mathrm{SI}$ is $95 \%$. 3 and Cariofilen oxide with $\mathrm{Mr}$ $=220$ and molecule formula $\mathrm{C}_{15} \mathrm{H}_{24} \mathrm{O}$, with percent SI is $97 \% 4$. There compounds are sequterpen $\left(\mathrm{C}_{15}\right)$, spesifik build from a head to tail isopren folowed "the isopren rule".

\section{Campound 5}

Was isolated from $n$-hexane fraction $\left(\mathrm{FD}_{2}\right)$ as yellow powder $(10.2 \mathrm{mg})$, gave a molecular ion at $\left[\mathrm{MH}^{+}\right]=603 / \mathrm{M}=602$, molecule formula $\mathrm{C}_{38} \mathrm{H}_{49} \mathrm{O}_{6}$. The IR spectrum characteristic showed strong bands for hydroxyl $\left(3537-3257 \mathrm{~cm}^{-1}\right)$ and both non conjugated $\left(1725 \mathrm{~cm}^{-1}\right)$ and conjugated (1637 $\mathrm{cm}^{-1}$ and $1600 \mathrm{~cm}^{-1}$ ) carbonyl groups. Melting point $133-135^{\circ} \mathrm{C}$. Analysis of the $1 \mathrm{D}$ and $2 \mathrm{D}$ NMR spectra in $\mathrm{C}_{3} \mathrm{D}_{6} \mathrm{O}$ with homo and hetero nuclear direct and long-range correlations allowed assignment of ${ }^{1} \mathrm{H}$ and ${ }^{13} \mathrm{C}$ NMR signals for compound $\mathbf{5}$ as listed in Table. 2, revealed signals assignable six vinylic methyl groups at $(\delta 1.49$; $1.60 ; 1.62 ; 1.63 ; 1.68$ and 1.69 ppm, $3 \mathrm{H}$ each singlet) and three vinyl proton at $(\delta 4.51 ; 4.93$; $5.02 \mathrm{ppm}, 1 \mathrm{H}$ each triplet) indicated contain of three isophrenyl groups. Two saturated methyls group appeared at $\delta(0.99$ and $1.17 \mathrm{ppm}, 3 \mathrm{H}$ each s) and were assigned methyl geminal group. (C-22 - C-23) correlated by HMBC two each other and to C-4; C-5 and C-6. It also showed two exomethylen protons at $(\delta 4.50$ and $4.53 \mathrm{ppm}$ each s) and one terminal methyl proton. An AMX system was evident from proton resonance at, $6.71(1 \mathrm{H}, d, J=7.30 \mathrm{~Hz}) ; 7.03(1 \mathrm{H}, d, J=7.35 \mathrm{~Hz})$; $7.20(s, 1 \mathrm{H})$ and typical ${ }^{13} \mathrm{C}$ NMR signals three substituted aromatic carbons at $\delta \mathrm{c} 149.5 ; 145.1$; 131.5 and conjugated carbonyl at $\delta c 196.5$, were indicated at 3,4 - deoxygenated benzoyl moiety. Resonance for six membered ring consisting non conjugated ketone at $\delta c$ 211.0. It shows the presence of three methine carbons of three substituted olefenic groups (- $\underline{\mathrm{CH}}=\mathrm{Me}$ x 3$)$ doublet at $125.9 ; 131.8$ and 132.3 and triplet at 112.1 for terminal methylene carbon $\left[\mathrm{C}(\mathrm{Me})=\underline{\mathrm{CH}}_{2}\right)$. The other assignment are at $132.9 ; 131.4$ and 132.3 ppm (C-19; C-26 and C-36; = $\underline{\mathrm{CMe}}$ ), 147.2 (C-36; $\left.=\underline{\mathrm{C}}=\mathrm{CH}_{2}\right)$. The compound $\mathbf{5}$ confirmed with literature (Fuller R.W. et al, 1999; Gustafson et al, 1992) and can be concluded that compound 5 is Guttiferon F. (The correlation HMBC of copound 5 list at Table 2. and Guttiferon F in Fig. 2).

\section{Antioxidant acivity}

The $n$-hexane, methanol, ethyl acetate, $n$ butanol extracts and Guttiferron F (compound 5) were screened for DPPH Radical scavenging activity, $n$-hexane, methanol, ethyl acetate, extracts and Guttiferron F (compound 5) are showed significant activity $\left(\mathrm{IC}_{50} 24.5,22.4,29.2\right.$, and 25.8 $\mu \mathrm{g} / \mathrm{mL}$ respectively) compare to the standard cuercetin ( $\mathrm{IC}_{50} 10.09 \mu \mathrm{g} / \mathrm{mL}$ ) and $n$-butanol extract showed moderate activity $\left(\mathrm{IC}_{50} 37,56 \mu \mathrm{g} / \mathrm{mL}\right)$. 
Table II. Chemical ship 'H, ${ }^{13}$ C NMR, HMQC and HMBC Data of Compound 5.

\begin{tabular}{|c|c|c|c|c|c|c|}
\hline \multirow{2}{*}{$\begin{array}{c}\text { No. } \\
\text { I }\end{array}$} & \multirow{2}{*}{$\begin{array}{c}{ }_{\text {I3C- }}{ }^{\text {NMR }(\delta,} \\
\text { Ppm) }\end{array}$} & \multirow{2}{*}{$\begin{array}{c}\text { HMQC } \\
\text { 'H (ס, ppm) } \\
-\end{array}$} & \multirow{2}{*}{$\begin{array}{l}\text { groups } \\
\mathrm{C}-\mathrm{OH}\end{array}$} & \multicolumn{3}{|c|}{ HMBC ${ }^{13} \mathrm{C}-\mathrm{NMR}(\delta, \mathrm{ppm})$} \\
\hline & & & & - & - & - \\
\hline 2 & 116.91 & - & C & - & - & - \\
\hline 3 & 211.00 & - & $C=O$ & - & - & - \\
\hline 4 & 69.00 & - & C & - & - & - \\
\hline 5 & 49.50 & - & C & - & - & - \\
\hline 6 & 47.60 & 1.45 & $\mathrm{CH}$ & C8 (48.88) & - & - \\
\hline 7 & 42.92 & $2.2(\mathrm{~d}, J=14.50 \mathrm{~Hz})$ & $\mathrm{CH}_{2}$ & C9 $(211.00)$ & - & \\
\hline 8 & 48.88 & - & c & - & - & - \\
\hline 9 & 211.00 & - & $C=O$ & - & - & - \\
\hline 10 & 196.50 & - & $C=O$ & - & - & - \\
\hline 11 & 131.46 & - & c & - & - & - \\
\hline 12 & 116.91 & $7.2(\mathrm{~s}, \mathrm{IH})$ & $\mathrm{C}-\mathrm{H}$ & $\mathrm{Cl} 3(145.1 \mathrm{I})$ & $\mathrm{Cl} 4(149.50)$ & $\mathrm{Cl} 6(125.09)$ \\
\hline 13 & $145.1 \mid$ & - & $\mathrm{C}-\mathrm{OH}$ & - & - & - \\
\hline 14 & 149.40 & & $\mathrm{C}-\mathrm{OH}$ & - & - & - \\
\hline 15 & 114.69 & $6.7 \mathrm{I}(\mathrm{d}, \mathrm{IH} J=7.30 \mathrm{~Hz})$ & $\mathrm{CH}$ & $\mathrm{Cl} 3(145.11)$ & & \\
\hline 16 & 125.09 & $7.03(\mathrm{~d}, \mathrm{IH} J=7.35 \mathrm{~Hz})$ & $\mathrm{CH}$ & $\mathrm{ClO}(196.5)$ & $\mathrm{ClI}(\mid 16.91)$ & CI5(II4.69) \\
\hline 17 & 26.59 & $2.7(\mathrm{~m})$ & $\mathrm{CH}_{2}$ & C3 $(211.00)$ & C9 $(211.00)$ & \\
\hline 18 & 125.89 & $4.93(t, \mathrm{IH})$ & $\mathrm{CH}$ & C20 (25.74) & C2I(I7.9) & \\
\hline 19 & 132.29 & - & C & - & - & - \\
\hline 20 & 25.74 & $1.62(s, 3 \mathrm{H})$ & $\mathrm{CH}_{3}$ & Cl8 (I25.89) & $\mathrm{Cl}$ (I32.29) & C2I (25.74) \\
\hline 21 & 17.90 & $\mathrm{I} .49(\mathrm{~s}, 3 \mathrm{H})$ & $\mathrm{CH}_{3}$ & $\mathrm{Cl}$ (I25.89) & $\mathrm{Cl9}(132.29)$ & C20 (25.74) \\
\hline 22 & 23.04 & 1.17 & $\mathrm{CH}_{3}$ & C5 (49.50) & C6 (47.60) & \\
\hline 23 & 27.33 & $0.99(s, 3 \mathrm{H})$ & $\mathrm{CH}_{3}$ & C4 (69.00) & C6 (47.60) & \\
\hline 24 & 27.21 & $2.01(\mathrm{~m})$ & $\mathrm{CH}_{2}$ & C25 (I24.03) & $\mathrm{C} 26(131.38)$ & - \\
\hline 25 & 124.03 & $5.02(t, \mathrm{IH})$ & $\mathrm{CH}$ & $C 26(131.38)$ & C27 (25.82) & \\
\hline 26 & 131.38 & - & c & - & - & - \\
\hline 27 & 25.82 & $1.63(s, 3 \mathrm{H})$ & $\mathrm{CH}_{3}$ & C2I (17.9) & C25 (124.03) & $C 28(18.18)$ \\
\hline 28 & 18.18 & $1.60(s, 3 \mathrm{H})$ & $\mathrm{CH}_{3}$ & C25 (I 24.03) & $\mathrm{C} 26(131.38)$ & C27 (25.82) \\
\hline 29 & 37.13 & $1.93(d d, \mathrm{~J}=12.85 ; 6.1)$ & $\mathrm{CH}_{2}$ & $\mathrm{Cl}(171.50)$ & & \\
\hline 30 & 44.40 & $2.69(\mathrm{~m}, \mathrm{l} .55 \mathrm{H})$ & $\mathrm{CH}$ & C32 (II2.05) & C35 (I22.07) & C9 $(211.00)$ \\
\hline 31 & $|50.3|$ & - & C & - & - & - \\
\hline 32 & | 12.05 & $4.5 \mathrm{I}(\mathrm{s}, \mathrm{IH}) ; 4.53(\mathrm{~s}, \mathrm{IH})$ & $\mathrm{CH}_{2}$ & C30 (44.40) & C33 (I8.38) & \\
\hline 33 & 18.38 & $1.59(s)$ & $\mathrm{CH}_{3}$ & C32 (II2.05) & & \\
\hline 34 & 32.75 & $2.02(m)$ & $\mathrm{CH}_{2}$ & C35 (I22.07) & C36 (I32.29) & \\
\hline 35 & 122.07 & $5.09(b, \mathrm{IH})$ & $\mathrm{CH}$ & C37 (18.12) & C38 (I7.9) & \\
\hline 36 & 132.29 & - & C & - & - & - \\
\hline 37 & 26.16 & $1.69(s, 3 \mathrm{H})$ & $\mathrm{CH}_{3}$ & C35 (122.07) & & \\
\hline 38 & 18.12 & $\mathrm{I} .68(\mathrm{~s}, 3 \mathrm{H})$ & $\mathrm{CH}_{3}$ & C35 (I22.07) & - & - \\
\hline
\end{tabular}




\section{CONCLUSION}

From the analysis with GC-MS of FA (essential oil) resulted a mixture of compounds Copaene $\left(\mathrm{C}_{15} \mathrm{H}_{24}\right)$ 1, 4,7-methanoazulene $\left(\mathrm{C}_{15} \mathrm{H}_{24}\right)$ 2, $\gamma$ - cadienene $\left(\mathrm{C}_{15} \mathrm{H}_{24}\right) \quad 3$ and Cariofilen oxide $\left(\mathrm{C}_{15} \mathrm{H}_{24} \mathrm{O}\right)$ 4. There compounds are sequterpen $\left(\mathrm{C}_{15}\right)$.

Compound 5. as yellow powder (10.2 mg), gave a molecular ion at $\left[\mathrm{MH}^{+}\right]=603 / \mathrm{M}=602$, melting point $133-135^{\circ} \mathrm{C}$, molecule formula $\mathrm{C}_{38} \mathrm{H}_{49} \mathrm{O}_{6}$. is Guttiferon F.

Antioxidant acivity of $n$-hexane, methanol, ethyl acetate, $n$-butanol extracts and Guttiferron $\mathrm{F}$ (compound 5) were screened for DPPH Radical scavenging activity, $n$-hexane, methanol, ethyl acetate, extracts and Guttiferron F (compound 5) are showed significant activity $\left(\mathrm{IC}_{50} 24.5,22.4\right.$, 29.2 , and $25.8 \mu \mathrm{g} / \mathrm{mL}$ respectively) compare to the standard cuercetin $\left(\mathrm{IC}_{50} 10.09 \mu \mathrm{g} / \mathrm{mL}\right.$ ) and $n$ butanol extract showed moderate activity $\left(\mathrm{IC}_{50}\right.$ $37,56 \mu \mathrm{g} / \mathrm{mL}$ ).

\section{ACKNOWLEDGMENT}

We are Thankful to Mr. Ismail Saleh Staff of Research Center for Biology -LIPI. Bogor for sampling and determine of specimen.

\section{REFERENCES}

Anne-Emmanuelle, H., Aumon, M.C., Mallet, S., Dumontet, V., Litaudon, M., Rodeau, D. and Richome P., 2004, Antioxidant from Garcinia veilardii, J Nat. Prod., 67, 707-709.

Balasubranian, K. and Rajagopalan, K., 1988, Xanthones from Garcinia mangostana, Structures of BR-Xanthone A and BRXanthone B, Phytochemistry, 27(5), I552I554.

Burkill, I.H., Birtwistle, W., Foxworthy, F.W., Scrivenor, J.B. and Watsan, J.G., 1935, A Dictionary of Economic Producds of the Malay Peninsula, Vol. I, Gaverments of the Straits Settlemens and Federated.

Chi-Kuan, H., Huang, Y-L. and Chen, C-C., 2002, Gracinone E, A Xanthone Derivtive, has Potent Cytotoxic Effect Againts Hepatocellular Carcinoma Cell Lines, Planta Medica, 68, 975 - 979.

Chen, S-X., Wan, M. and Loh, B-N., 1996, Active Constituens Against HIV-I Protease from Garcinia mangostana, Planta Med., 62, 381382.
Fuller, R.W., John, W. Blunt, Jamie L. B., John H., Cardellina H. and Michael R. Boyd (1999), Guttiferron F, the First Prenylated Benzophenone from Allablackia stuhlmannii, J. Nat. Prod., 62, $130-132$.

Gustafson, K.R., John, W.B., Murray, H.G.M., Richard, W.F., Tawnya, C. McKee, John, H.C., HMcMahon, J.B., Cragg, G.M. and Michael, R.B., 1992, The Guttiferones, HIV Inhibitory Benzophenones from, Tetrahedron, 48(46), 10092-10102.

Inuma, M., Tosa, H., Ito, T., Tanaka, T. and Riswan S., 1996, Three New BenzophenoneXanthone Dimers from the Root of Garcinia dulcis, Chem. Pharm. Bull., 44(9), 1744-1747.

Liao, C-H., Ho, C-T. and Lin, J-K., 2005, Effects of Garcinol on Free Radical Generation and NO Production in Embryonic Rat Cortical Neuron and Astrocytes, Biochem. and Biophsy. Res. Comm., 329, I306-13/4.

Likhitwitauwuid, K., Phadungcharoen, T. and Krungkrai, J., 1998, Antimalarial Xanthones from Garcinia cowa, Planta Medica, 64, 70-72.

Likhiawitayawuid, K., Chanmahasthien, W., Nijsiriruangrungsi and Krungkrai, J., 1998, Xanthone with Antimalarial Activity from Garcinia dulcis, Planta Medica, 64, 28I-282.

Molyneux, P., 2004, The Use of the stable free radical diphenylpicrylhydrazyl (DPPH) for estimating antioxidant activity, Songklanakarin J. Sci. Technol., 26(2), 2II219.

Odile, T., Fahy, J., Dumotet, V., Chiaroni, A., Riche, C., Tri, M.V. and Sěvenet T., 2000, Cytotoxic Prenylxanthone from Garcinia bracteata, J. Nat. Prod., 63(4), 44I -446).

Permana, D., Nordin, H.J. Lajis, Mukram, M. Mackeen, Abdul M. Ali, Norio Aimi, Kitajima M. and Takayama H., 200I, Isolation and Bioactive of Constituents of the Roots of Garcinia atroviridis, J. Nat. Prod., 64, 976-979.

Quan-Bin, H., Lee, S-F, Qiau, C-P, He, Z-D, JinZheng, Sun, H-D. and $\mathrm{Xu}, \mathrm{H}-\mathrm{X}$., 2005, Complete NMR Assignments of The Antibacterial Biflavonoid GBI from Garcinia kola, Chem. Pharm. Bull, 53(8), 1034-1036.

Quan-Bin, H., Wang, Y-L., Ling Yang, Tso, T-F, Qiao, C-F, Song, J-Z, Xu, L-J, Chen, S-L, Yang, D-J. and Xu H-X., 2006, Cytotoxic Polyphrenilated Xanthone from the Resin 
of Garcinia hanburyi, Chem. Pharm. Bull, 54(2), 265-267.

Sudpondma, Y., Rukachaisirkul, V. and Phongpaichit, S., 2005, Antibacterial Cagec-Tetraprenylated Xanthones from fruit Garcinia hanburyi, Chem. Pharm. Bull, 53(7), 850-852.

Suksamrarn, S., Suwanapoch, N., Phakhodee, W., Thauhiranlert, J., Ratananukul, P., Chimnoi, N. and Suksamsarn, A., 2003, Antibacterial Activity of Prenylated Xanthones from the Fruits of Garcinia mangostana, Chem. Pharm. Bull, 5I(7), 857-859.

Suksamrarn, S., Komutiran, O., Ratananukul, P., Chimnoi, N., Lartpornmatulee, N. and Suksamrarn, A., 2006, Cytotoxic Prenylated Xanthones from the Young Fruit of Garcinia mangostana, Chem. Parm. Bull, 54(3), 30I-305.

Terashima, K., Takaya, Y. and Niwa, M., 2002, Powerful Antioxydative Agent Based on Garcionic Acid from Garcinia kola, Bioorg. Med. Chem., 10, 1619-1625.

Tona, L., et al., 2004, In vitro Antiplasmodial Activity of Extracts and Fraction from
Seven Medicinal Plants Use in The Democratic Republic of Congo, J. of Ethno. Pharm. 93, 27-32.

Vatcharin, R., Todpetch, K., Watthanaphanit, A, Saengsanae, N. and Phongpaichit, S., 2005, An Antibacterial Biphenyl Derivative from Garcinia bancana Miq, J. Nat. Prod., 68, 1218-1221.

Vatcharin, R., Pharm, W., Sudpindma, Y. and Phongpaichit, S., 2005, An Antibacterial Biphenyl Derivative from Garcinia bancana Miq., Chem. Pharm. Bull., 53(3), 342-343.

Withmore, M.A., 1973, Tree forest of Malaya. Malaysia Forest Department, Ministry of Primary Industries, Logman. P. 206.

Xu, Y-J., S. C.Yip, Kosela, S., Fitri, E., Hanafi, M., Goh, S.H. and Sim, K.Y., (2000), Novel Cytotoxic, Polyphrenylated Heptacyclic Xanthonoids from Indonesian Garcinia gaudichaudii (Guttiferae), Org. Let., 2 (24), 3945-3948.

Yamaguchi, F., Saito, M.O., Ariga, T., Yoshimura, Y. and Nakazawa, H., 2000, Free Radical Scavenging Activity and Antiulcer Activity from Garcinia indica Fruit Rind, J. Agric. Food Chem., 48, 2320-3225. 